\title{
GUARANTEEING ASYMPTOTIC ZERO INTERSAMPLING TRACKING ERROR VIA A DISCRETIZED REGULATOR AND EXPONENTIAL HOLDER FOR NONLINEAR SYSTEMS
}

\author{
B. Castillo-Toledo \& G. Obregón-Pulido. \\ Centro de Investigación y de Estudios Avanzados del IPN, Unidad Guadalajara A.P. 31-438, Plaza La Luna, 44550, Guadalajara, Jal.
}

Received May $5^{\text {th }} 2001$ and accepted October $10^{\text {th }} 2002$

\section{ABSTRACT}

In this paper we present the design of a robust discretized controller based in the theory of regulation for nonlinear systems. In the general command tracking and disturbance rejection problem, it is known that a sample data controller using zero order holder may only guarantee asymptotic tracking at the sampling instances. We show that by means of a so-called exponential holder, we are able to guarantee asymptotic zero output tracking error, not only in the sampling instant, but also between these instant. Some considerations on the design of exponential holders are presented. The application in simulation to some typical nonlinear systems shows the good performance of the proposed scheme.

KEYWORDS: Nonlinear Systems, Discrete time control, Regulation Theory.

\section{INTRODUCTION}

A central problem in the control theory is that of manipulating the inputs of a system in such a way that the outputs of that system tracks, at least asymptotically, a defined reference signals, preserving at the same time some desired stability property of the close-loop system. More precisely, consider the nonlinear system described by

$$
\begin{gathered}
\dot{x}=f(x, u, w, \theta) \\
\dot{w}=s(w) \\
e=h(x, w, \theta),
\end{gathered}
$$

where $\theta \in \mathfrak{R}^{l}$ is a parameter vector, $u \in \mathfrak{R}^{m}$ is the input signal, $x \in \mathfrak{R}^{n}$ is the state of the system, $w \in \mathfrak{R}^{p}$ represents the state of an external signal generator, described by (2), which provides the reference and/or perturbation signals. Equation (3) describes the output tracking error $e \in \mathfrak{R}^{q}$ defined as the difference between the system output and the reference signal.

For this system, the mentioned problem has been treated under different approaches, among which is the regulator theory. In general terms, this problem consists in finding a submanifold (the steady state submanifold) on which the output tracking error is zeroed, as well as an input signal (the steady state input) which makes this submanifold 
invariant and attractive. The regulator problem has been studied intensively both in the linear case and recently in the nonlinear setting $[2-3,10]$. In [4-7, 9], for the linear case, a solution of the problem was given in terms of the solution of certain algebraic matrix equations (Francis equations), which describe the existence of the invariant submanifold aforementioned.

For the nonlinear case, Isidori [1] has extended the previous ideas by showing that the respective nonlinear regulator problem is solved by means of the solution of partial differential equations, named Francis-Isidori-Byrnes (FIB) equations. Moreover, along the same lines of [7], it has been shown that the inclusion of an internal model in the controller structure is necessary and sufficient for the robust regulation [8]. In the same direction, Isidori, Byrnes and Delli Priscolli $[2-3,10]$, have presented a robust error feedback controller which relies also on the existence of an internal model, representing the inclusion of the exosystem dynamics into an observable one, which allows to generate, as in the linear case, all the possible steady state inputs for the admissible values of the system parameters. Basically, the Robust Regulator Problem (RRP) consist in finding a dynamic controller

$$
\begin{gathered}
\dot{\xi}=\varphi(\xi, e) \\
u=\vartheta(\xi)
\end{gathered}
$$

such that, for all admissible parameter values in a suitable neighborhood $P$ of the nominal parameter vector, the following condition hold:

CS) The equilibrium point $(x, \xi)=(0,0)$ of the system

$$
\begin{aligned}
& \dot{x}=f(x, \vartheta(\xi), 0, \theta) \\
& \dot{\xi}=\varphi(\xi, h(x, 0, \theta))
\end{aligned}
$$

is asymptotically stable

CR) The solution of close-loop system (6) satisfies that

$$
\lim _{t \rightarrow \infty}\|e(t)\|=0
$$

A complete solution to this problem has been presented in [1, $2 \& 3$ ]. Roughly speaking, this solution is based on the existence of nonlinear mappings $x_{s s}=\pi(w, \theta)$ and $\xi_{s s}=\sigma(w, \theta)$ that satisfy the so-called Francis-Isidori-Byrnes (FIB) equations

$$
\begin{aligned}
& \frac{\partial \pi(w, \theta)}{\partial w} s(w)=f(\pi(w, \theta), \vartheta(\sigma(w, \theta)), w, \theta) \\
& \frac{\partial \sigma(w, \theta)}{\partial w} s(w)=\varphi(\sigma(w, \theta), 0) \\
& 0=h(\pi(w, \theta), w, \theta)
\end{aligned}
$$

for all values of $\theta$ in $P$. To obtain a solution of RRP, we consider the linear approximation of the system (1)-(5). 


$$
\begin{aligned}
& \dot{x}=A_{0} x+B_{0} u+P_{0} w \\
& \dot{w}=S w \\
& e=C_{0} x+Q_{0} w \\
& \dot{\xi}=F \xi+G e \\
& u=H \xi
\end{aligned}
$$

where

$$
\begin{aligned}
& A_{0}=\left.\frac{\partial f(x, u, w, \theta)}{\partial x}\right|_{(0,0,0,0)} ; B_{0}=\left.\frac{\partial f(x, u, w, \theta)}{\partial u}\right|_{(0,0,0,0)} ; P_{0}=\left.\frac{\partial f(x, u, w, \theta)}{\partial w}\right|_{(0,0,0,0)} \\
& C_{0}=\left.\frac{\partial h(x, w, \theta)}{\partial x}\right|_{(0,0,0)} ; Q_{0}=\left.\frac{\partial h(x, w, \theta)}{\partial w}\right|_{(0,0,0)} ; S=\left.\frac{\partial s(x)}{\partial w}\right|_{(0)} ; F=\left.\frac{\partial \varphi(\xi, e)}{\partial \xi}\right|_{(0,0)} \\
& G=\left.\frac{\partial \varphi(\xi, e)}{\partial e}\right|_{(0,0)} ; H=\left.\frac{\partial \vartheta(\xi)}{\partial \xi}\right|_{(0)}
\end{aligned}
$$

Proposition 1: [1, 2, 3 \& 10] Assume the following assumptions hold:

$\mathrm{H1})$ The pair $\left(\mathrm{A}_{0}, \mathrm{~B}_{0}\right)$ is stabilizable.

$\mathrm{H} 2)$ The pair $\left(A_{0}, C_{0}\right)$ is detectable.

Then, the robust output regulation problem can be solved by a linear controller, if for all $\theta \in \rho$, there exist mappings $x_{s s}=\pi(w, \theta), u_{s s}=\gamma(w, \theta)$, with $\pi(0, \theta)=0, \gamma(0, \theta)=0$, defined in a neighborhood of the origin of $(x, w)=(0,0)$, satisfying the conditions

$$
\begin{aligned}
& \frac{\partial \pi(w, \theta)}{\partial w} s(w)=f(\pi(w, \theta), \gamma(w, \theta), w, \theta) \\
& 0=h(\pi(w, \theta), w, \theta)
\end{aligned}
$$

and for each $\mathrm{i}=1,2, . . \mathrm{m}$, there is a set of real numbers $a_{0}{ }^{i}, a_{1}{ }^{i}, a_{2}{ }^{i}, \ldots, a_{r i-1}{ }^{i}$, such that the steady state input $\gamma_{i}(w, \theta)$ satisfies

$$
L_{s}^{r i} \gamma_{i}(w, \theta)=a_{0}^{i} \gamma_{i}(w, \theta)+\ldots+a_{r i-1}{ }^{i} L_{s}{ }^{r i-1} \gamma_{i}(w, \theta)
$$

and the matrix

$$
\left[\begin{array}{cc}
A_{0}-\lambda & B_{0} \\
C_{0} & 0
\end{array}\right]
$$

is nonsingular for every $\lambda$ which is a root of any of the polynomial 


$$
p(\lambda)=a_{0}{ }^{i}+a_{1}{ }^{i} \lambda+a_{2}{ }^{i} \lambda^{2}+\ldots a_{r i-1}{ }^{i} \lambda^{r i-1}-\lambda^{r i}
$$

Remark 1: The mapping $x_{s s}=\pi(w, \theta)$ is the steady state submanifold where the error is zeroed, and $\left.u_{s s}=\gamma(w, \theta)\right\}$ is the steady state input which makes invariant the steady state submanifold. Condition (10) signifies the fact that the steady state input can be generated independently from the values of the parameters, by the linear dynamical system

$$
\begin{aligned}
& \dot{z}=\Phi z \\
& u_{s s}=\tilde{H} z
\end{aligned}
$$

where

$$
\begin{aligned}
z & =\left[\begin{array}{llll}
z_{1} & z_{2} & \cdots & z_{m}
\end{array}\right]^{T} ; \\
z_{i} & =\left[\begin{array}{llll}
\gamma_{i}(w, \theta) & L_{s} \gamma_{i}(w, \theta) & \cdots & L_{s}^{r i-1} \gamma_{i}(w, \theta)
\end{array}\right]^{T} ; \\
H & =\left[\begin{array}{cccc}
H_{1} & 0 & \cdots & 0 \\
0 & H_{2} & \cdots & 0 \\
\vdots & \vdots & \ddots & \vdots \\
0 & 0 & \cdots & H_{m}
\end{array}\right] ; H_{i}=\left[\begin{array}{llll}
1 & 0 & \cdots & 0
\end{array}\right]_{1 \times r i} .
\end{aligned}
$$

and

$$
\Phi=\operatorname{diag}\left(\Phi_{1}, \Phi_{2}, \ldots, \Phi_{m}\right) ; \quad \Phi_{i}=\left[\begin{array}{ccccc}
0 & 1 & 0 & \cdots & 0 \\
0 & 0 & 1 & \cdots & 0 \\
\vdots & \vdots & \vdots & \ddots & \vdots \\
0 & 0 & 0 & \cdots & 1 \\
a_{o}^{i} & a_{1}^{i} & a_{2}^{i} & \cdots & a_{r i-1}{ }^{i}
\end{array}\right]
$$

The dynamic controller may be viewed as a combination of two systems: one that stabilizes the system and another one that provides the steady state input on the steady state submanifold. In fact the controller can be constructed considering the linear approximation of the closed loop system. Let us take

$$
\tilde{x}=x-\pi(w, \theta)
$$

and derive with respect to the time, so that

$$
\begin{aligned}
& \dot{\tilde{x}}=f(\tilde{x}+\pi(w, \theta), u, w, \theta)-\frac{\partial \pi}{\partial w} s(w) \\
& \dot{\tilde{x}}=f(\tilde{x}+\pi(w, \theta), u, w, \theta)-f(\pi(w, \theta), \gamma(w, \theta), w, \theta)
\end{aligned}
$$

Since the steady state $\gamma(w, \theta)$ is generated by the system (12), we substitute $\gamma(w, \theta)$ by $\tilde{H} z$, so that equation (13) together with (12) gives 


$$
\begin{aligned}
& \dot{\tilde{x}}=f(\tilde{x}+\pi(w, \theta), u, w, \theta)-f(\pi(w, \theta), \tilde{H} z, w, \theta) \\
& \dot{z}=\Phi z \\
& e=h(\tilde{x}+\pi(w, \theta), w, \theta)
\end{aligned}
$$

We may rewrite this equation considering its linear approximation as

$$
\begin{gathered}
\dot{\tilde{x}}=A_{0} \tilde{x}-B_{0} \tilde{H} z+B_{0} u+f_{2}(\tilde{x}+\pi(w, \theta), u, w, \theta)-f_{2}(\pi(w, \theta), \tilde{H} z, w, \theta) \\
\dot{z}=\Phi z \\
e=C_{0} \tilde{x}+C_{0} \pi(w, \theta)+\tilde{h}(\tilde{x}+\pi(w, \theta), w, \theta)
\end{gathered}
$$

then, if $\mathrm{H} 1$ and $H 2$ hold, we can construct the robust controller [2]

$$
\begin{aligned}
& {\left[\begin{array}{l}
\dot{\xi}_{1} \\
\dot{\xi}_{2}
\end{array}\right]=\left[\begin{array}{cc}
A_{0}-G_{0} C_{0}+B_{0} K & 0 \\
-G_{1} C_{0} & \Phi
\end{array}\right]\left[\begin{array}{l}
\xi_{1} \\
\xi_{2}
\end{array}\right]+\left[\begin{array}{l}
G_{0} \\
G_{1}
\end{array}\right] e(t)} \\
& u=K \xi_{1}+\tilde{H} \xi_{2}=H \xi
\end{aligned}
$$

where $K$ and $\mathrm{G}_{0}, \mathrm{G}_{1}$, make $\left(A_{0}+B_{0} K\right)$ and $\left(\begin{array}{cc}A_{0}-G_{0} C_{0} & -B_{0} \tilde{H} \\ -G_{1} C_{0} & \Phi\end{array}\right)$ Hurwitz, respectively. To verify that equations (7) are satisfied, we choose $x_{s s}=\pi(w, \theta)$ and $\xi_{s s}=\sigma(w, \theta)=\left[\begin{array}{ll}0 & \sigma_{2}(w, \theta)\end{array}\right]^{T}$ where

$$
\sigma_{2}(w, \theta)=\left[\begin{array}{llll}
\gamma_{1}(w, \theta) \cdots & L_{s}{ }^{r_{1}-1} \gamma_{1}(w, \theta) \cdots & \gamma_{m}(w, \theta) \cdots & L_{s}{ }^{r_{m}-1} \gamma_{m}(w, \theta)
\end{array}\right]^{T}
$$

An interesting point which arises here is if it is possible to construct a discrete controller in such a way that the properties of stabilization and tracking for continuous system are maintaining. Several works have been done in this direction [11 - 14]. In particular, in [11] a discrete regulator combined with a particular class of holder, named exponential holder was presented for the case of linear systems. In this paper, we discuss how to solve the respective discretized regulation problem for nonlinear systems. <?xml:namespace prefix $=0 \mathrm{~ns}=$ "urn: schemas-microsoftcom:office:office" />

\section{A DISCRETIZED REGULATOR}

In order to implement a discretized controller we take the discretization of (14)-(16), which may be written as

$$
\begin{aligned}
& \tilde{x}_{k+1}=e^{A_{0} T} x_{k}-\int_{k T}^{k T+T} e^{A_{0}(k T+T-\tau)} B_{0} \tilde{H} z(\tau) d \tau+\int_{k T}^{k T+T} e^{A_{0}(k T+T-\tau)} B_{0} u(\tau) d \tau+\phi\left(\tilde{x}_{k}, w_{k}, z_{k}\right) \\
& z_{k+1}=e^{\Phi T} z_{k} \\
& e_{k}=h\left(\tilde{x}+\pi\left(w_{k}, \theta\right), w_{k}, \theta\right)
\end{aligned}
$$


or in the matrix form

$$
\begin{aligned}
& {\left[\begin{array}{l}
\tilde{x}_{k+1} \\
z_{k+1}
\end{array}\right]=\left[\begin{array}{cc}
A_{d} & -\Lambda \\
0 & \Phi_{d}
\end{array}\right]\left[\begin{array}{l}
\tilde{x}_{k} \\
z_{k}
\end{array}\right]+\left[\begin{array}{c}
\tilde{u} \\
0
\end{array}\right]+\left[\begin{array}{c}
\phi\left(\tilde{x}_{k}, w_{k}, z_{k}\right) \\
0
\end{array}\right]} \\
& e_{k}=h\left(\tilde{x}_{k}+\pi\left(w_{k}, \theta\right), w_{k}, \theta\right) \\
& e_{k}=C_{0}\left(\tilde{x}_{k}+\pi\left(w_{k}, \theta\right)\right)+\tilde{h}\left(\tilde{x}_{k}+\pi\left(w_{k}, \theta\right), w_{k}, \theta\right)
\end{aligned}
$$

where

$$
\begin{aligned}
& A_{d}=e^{A_{0} T} ; \quad \Lambda=\int_{0}^{T} e^{A_{0}(T-\delta)} B_{0} \tilde{H} e^{\Phi \delta} d \delta ; \quad 0 \leq \delta<T \\
& \Phi_{d}=e^{\Phi T} ; \tilde{u}=\int_{0}^{T} e^{A_{0}(T-\delta)} B_{0} u(k T+\delta) d \delta .
\end{aligned}
$$

A central issue in the implementation of the robust controller is the exponential term in (20). As far as this term is continuous, we give in this section some guidelines for constructing such device. Let us consider the matrix $\Phi$ given by (12). This matrix is the composition of $m$ similar subsystems, namely:

$$
\Phi=\operatorname{diag}\left(\Phi_{1}, \Phi_{2}, \ldots, \Phi_{m}\right) ; \quad \Phi_{i}=\left[\begin{array}{ccccc}
0 & 1 & 0 & \cdots & 0 \\
0 & 0 & 1 & \cdots & 0 \\
\vdots & \vdots & \vdots & \ddots & \vdots \\
0 & 0 & 0 & \cdots & 1 \\
a_{o}^{i} & a_{1}^{i} & a_{2}{ }^{i} & \cdots & a_{r i-1}{ }^{i}
\end{array}\right]
$$

At this point, we may design a circuit that reproduces directly the equation $\tilde{H} e^{\Phi \delta} \xi_{2 k}$ by means of integrators and gains, where each integrator take the initial condition of the discrete controller, as is shown in Figure 1.

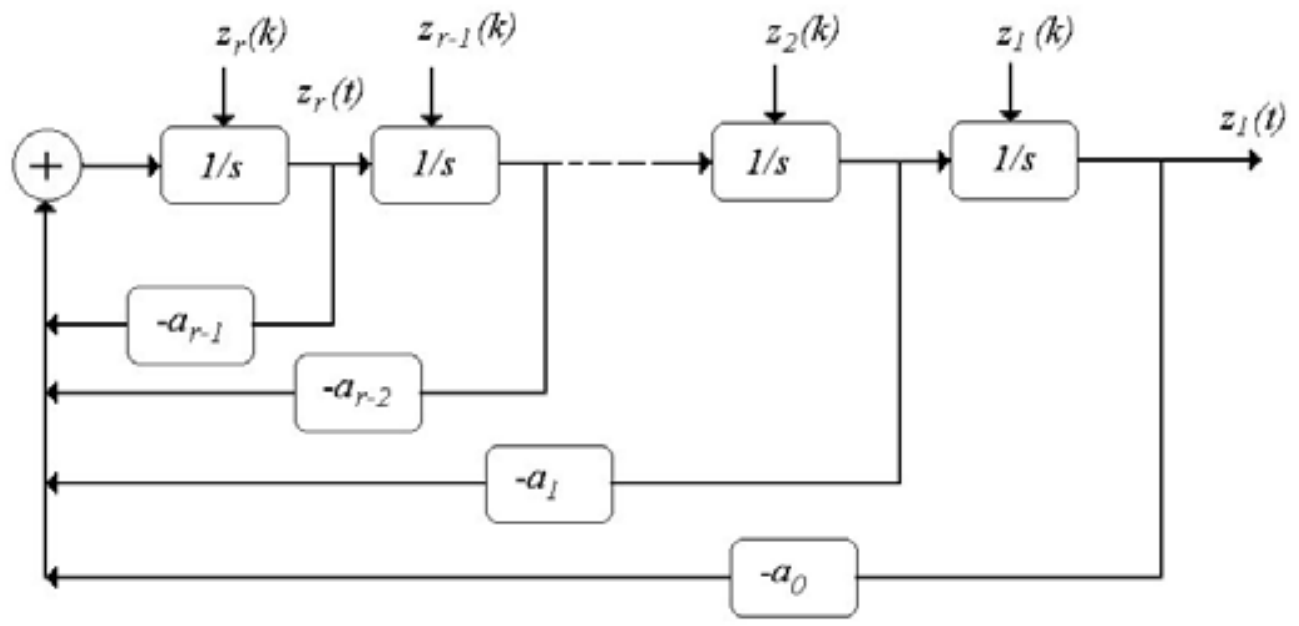

Figure 1. Implementation of the immersion by integrators.

This is possible since the solution to equation (12) for each subsystem is given by

$$
z_{i}(t)=e^{\Phi_{i}\left(t-t_{0}\right)} z\left(t_{0}\right) \quad i=1 \ldots m
$$


that can be implemented by means of integrators with variable initial conditions, for which a realization may be obtained as in Figure 2.



Figure 2. Integrator with variable initial condition.

This circuit is governed by

$$
\begin{gathered}
V_{0}\left(t_{1}+t\right)=V_{0}\left(t_{1}\right) e^{-\frac{t}{R C}}+\left(e^{-\frac{t}{R C}}-1\right) V_{i}\left(t_{1}\right) \\
\text { for pulse }=1 \text { and } t_{i}=\text { Instant in which pulse }=1 \\
V_{0}\left(t_{2}+t\right)=V_{0}\left(t_{2}\right)-\frac{1}{R_{3} C} \int_{t_{2}}^{t} V_{i}(\tau) d \tau
\end{gathered}
$$

for pulse $=0$ and $t_{2}=$ Instant in which pulse $=0$.

where $R_{1}=R_{2}=R$. When pulse $=1$, the operational amplifier takes the Inversor Low Pass filter configuration and the output takes the value $-V_{1}\left(t_{1}\right)$ after $5 R C$ seconds. On the other hand, when pulse $=0$, the operational amplifier changes to the integrator configuration with initial condition $V_{o}\left(t_{2}\right)=-V_{(}\left(t_{1}\right)$. ( See [18] for more details).

For example, for the sinusoidal generator

$$
\begin{aligned}
& \dot{\eta}_{1}=\eta_{2} \\
& \dot{\eta}_{2}=-\alpha^{2} \eta_{1}
\end{aligned}
$$


with $\alpha=2$; a circuit realization is shown in Figure 3 .

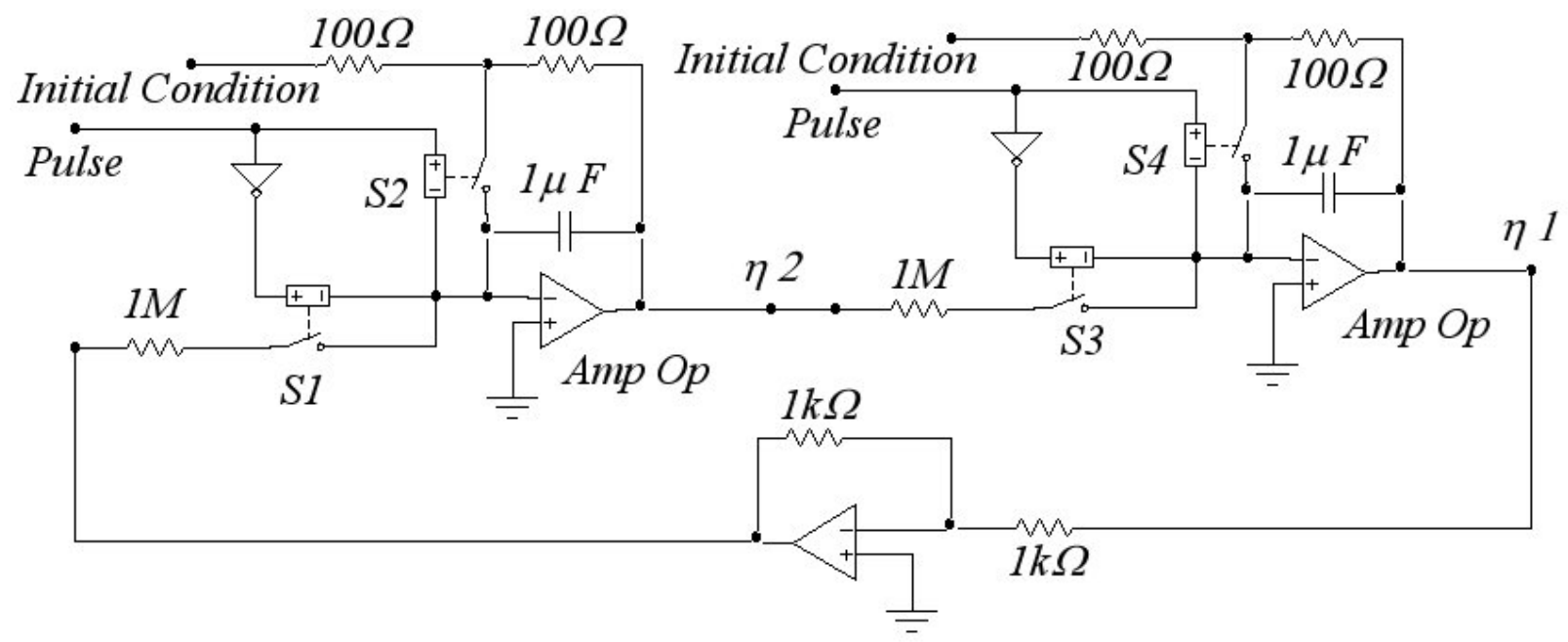

Figure 3. Exponential hold for a sinusoidal signal.

In this circuit the constant time is $R C=0.0001 \mathrm{~s}$, which implies that in $0.5 \mathrm{~ms}$ the desired initial condition is reached. The response of this circuit for initial condition given by $\eta_{1}=0, \eta_{2}=2$ is given in Figure 4 .

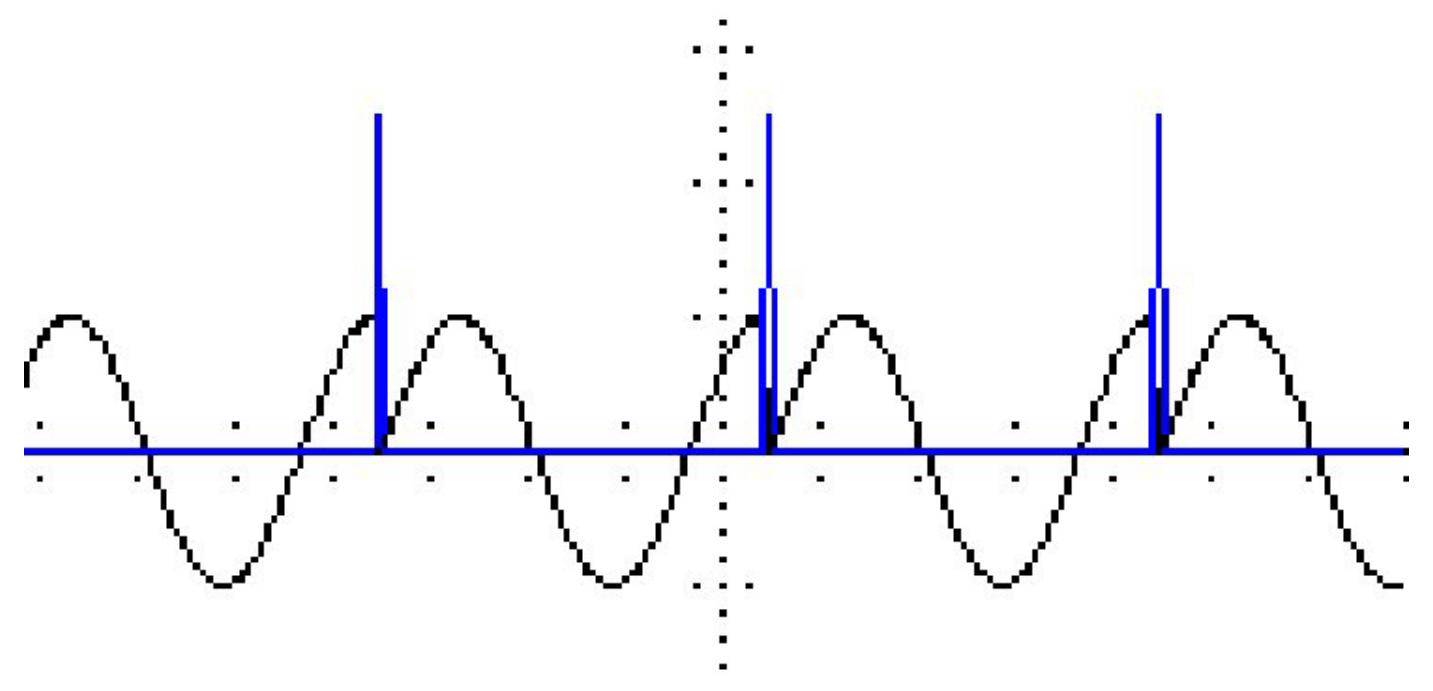

Figure 4. Response of the exponential holder.

\section{SOME ILUSTRATIVE EXAMPLES.}

Example 1. Suppose we have a DC motor whose inductance is neglected, described by

$$
\begin{aligned}
& \dot{x_{1}}=x_{2}+w_{1}+w_{2} \\
& \dot{x_{2}}=a_{0} x_{1}+b_{0} u-w_{1}-w_{2} \\
& y=x_{1}+w_{3}+w_{4}
\end{aligned}
$$


where $w_{1}=5 \sin (5 t), w_{2}=5 \cos (5 t), w_{3}=0.5 \sin (t), w_{4}=0.5 \cos (t), y_{\text {ref }}=w_{3}, a_{0}=-0.5, b_{0}=0.25$.

If we construct the robust discretized regulator considering only a zero order holder the output tracking error in continuous time is not zeroed, even when the discrete error is, as can be seen in Figures 5 and 6 . The discretized controller with exponential holder overcomes this situation, as shown in Figures 7 and 8, where some parameters variations has been introduced. We see that this controller is effective to compensate such variations.



Figure 5. Error signal in continuous time

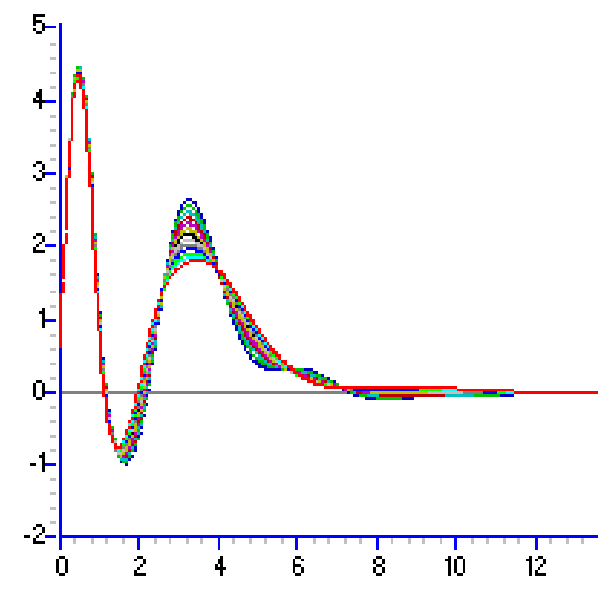

Figure 7. Error signals for a $\pm 25 \%$.

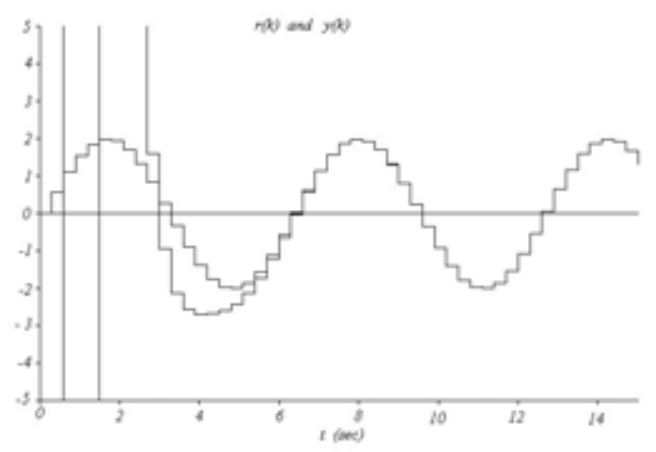

Figure 6. Output and reference signals in discrete time.

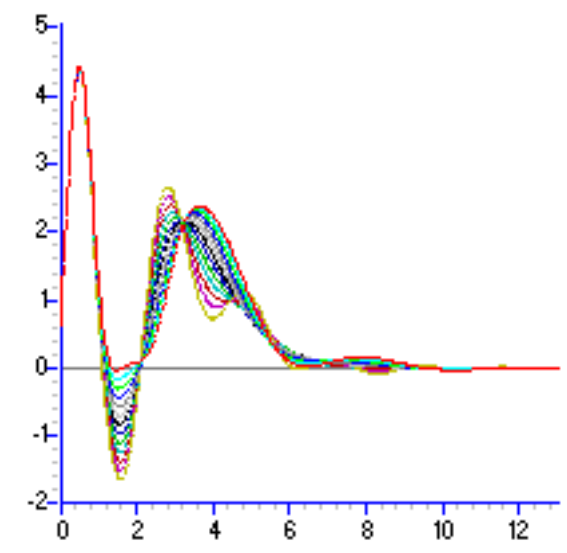

Figure 8. Error signals for $b_{\circ} \pm 12 \%$.

Example 2. Consider the Vander der Pol oscillator given by

$$
\begin{aligned}
& \dot{x}_{1}=x_{2} \\
& \dot{x}_{2}=-x_{1}+\theta x_{2}-x_{2}{ }^{3}+u
\end{aligned}
$$

with the exosystem

$$
\begin{aligned}
& \dot{w_{1}}=w_{2} \\
& \dot{w_{2}}=-w_{1}
\end{aligned}
$$

The output tracking error is defined as $e^{=} x_{i}-W_{i}$ for which the steady state submanifold $\Pi(W, \theta)$, and the steady state input $\gamma(W, \theta)$ are 


$$
\begin{aligned}
& \pi_{1}(w, \theta)=w_{1} \\
& \pi_{2}(w, \theta)=w_{2} \\
& \gamma(w, \theta)=w_{2}^{3}-\theta w_{2}
\end{aligned}
$$

The system satisfies condition (10), since

$$
\begin{aligned}
& z_{1}=\gamma(w, \theta)=w_{2}{ }^{3}-\theta w_{2} \\
& \dot{z}_{1}=z_{2}=-3 w_{1} w_{2}{ }^{2}+\theta w_{1} \\
& \dot{z_{2}}=z_{3}=-3 z_{1}-2 \theta w_{2}+6 w_{1}{ }^{2} w_{2} \\
& \dot{z_{3}}=z_{4}=-7 z_{2}+6 \theta w_{1}-6 w_{1}{ }^{3} \\
& \dot{z}_{4}=-9 z_{1}-10 z_{3}
\end{aligned}
$$

so the steady state input can be generated by the system

$$
\dot{z}=\left[\begin{array}{cccc}
0 & 1 & 0 & 0 \\
0 & 0 & 1 & 0 \\
0 & 0 & 0 & 1 \\
-9 & 0 & -10 & 0
\end{array}\right] z
$$

which is linear. The performance of the robust discretized regulator with exponential holder, are shown in Figure 9 , where the output tracking error is displayed for several values of the parameter $\theta$.

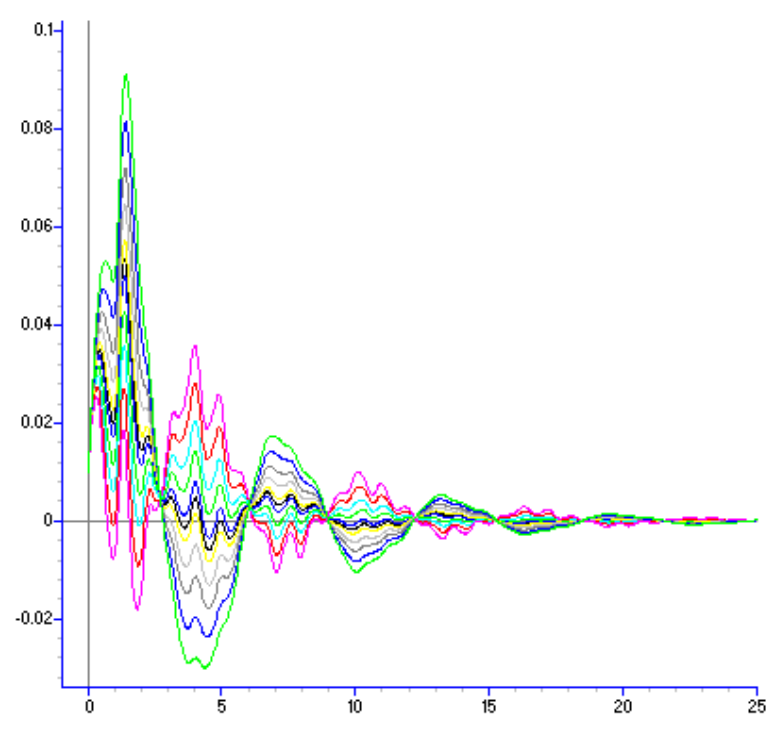

Figure 9. Error signal for $-0.2<\theta<0.2$. 


\section{CONCLUSIONS}

In this work, we propose a method for constructing a robust discretized controller for nonlinear systems, by means of a so-called exponential holder. This device allows one to construct a controller, which combines a discrete subsystem, and a continuous one, to guarantee zero output tracking error not only at the sampling instant, but also in the intersampling period. We discuss briefly how it is possible to construct such exponential holder, and show, by examples, the performances of the proposed control scheme. It is shown that the scheme proposed provides a combination of a digital controller with continuous devices allowing the improvement of the closed-loop response of a control system.

\section{ACKNOWLEDGEMENT}

Work supported partially by CONACyT under grant 37687A.

\section{REFERENCES}

[1] Isidori A., Nonlinear Control Systems, 3th Ed., Springer Verlag, 1998.

[2] Byrnes C. I., Delli Priscoli F. \& Isidori A., Output regulation of uncertain nonlinear systems, Birkhauser, 1997.

[3] Byrnes C. I., Delli Priscolli F., Isidori A. \& Kang W., "Structurally Stable Output Regulation of Nonlinear Systems", Automatica, Vol 33, 1997, pp. 369-385.

[4] Davison E. J. \& Goldenberg A., "The robust control of a general servomechanism problem: the servo compensator", Automatica, Vol 11, 1975, pp. 461-471.

[5] Sebakhy O. A. \& Wonham W. M., "Design procedure for multivariable regulator", Automatica, Vol. 12, 1976, pp. 467-478.

[6] Francis B. A., "The Internal Model principle of control theory", Automatica, Vol. 12, 1976, pp. 457-465.

[7] Francis B. A., "The linear multivariable regulator problem", SIAM J. Control Optimiz., Vol. 15, 1977, pp. 486-505.

[8] Hepburn J. S. A. \& Wonham W. M., "Error feedback and internal model on differentiable manifolds", IEEE Trans. Aut. Control, Vol. 29, 1984, pp. 397-403.

[9] Wonham W. M., Linear Multivariable Control: A Geometric Approach, 2nd Ed. New-York: Springer-Verlag, 1979.

[10] Isidori A. and Byrnes C. I. "Output regulation of nonlinear systems", IEEE Trans. Aut. Control, Vol. 35, No. 2, 1990, pp. 963-972.

[11] Yung-Chun W. \& Nie-Zen-Yen, "A Ripple Free Sample-Data robust Servomechanism Controller Using Exponential Hold", IEEE Trans. Aut. Control, Vol. 39, No. 6, 1994, pp. 1287-1291.

[12] Franklin G. F. \& Emami-Naeini A., "Design of Ripple Free Multivariable Robust Servomechanism", IEEE Trans. Aut. Control, Vol. AC-31, No. 7, 1986, pp. 661-664.

[13] Castillo-Toledo B., Di Gennaro S., Monaco S. \& Normand- Cyrot, "On Regulation Under Sampling", IEEE Trans. Aut. Control, Vol. 42, No. 6, 1997, pp. 864-868.

[14] Kabamba P. T., "Control of Linear Systems Using Generalized Sample-Data Hold Functions", IEEE Trans. Aut. Control, Vol. AC-32, No. 9, 1987, pp. 772-782. 


\section{Authors Byography}

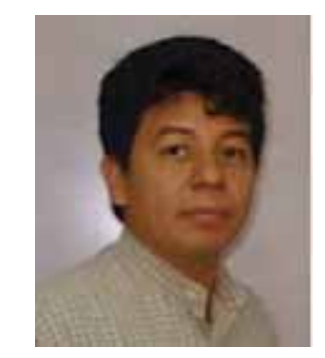

\section{Bernardino Castillo-Toledo}

Dr. Castillo-Toledo was born in Cd. Ixtepec, Oaxaca, México in 1959. He received the B. Sc. degree in Electrical Engineering from the National Polytechnic Institute (IPN), the M. Sc. Degree from the Center of Research and Advanced Studies (CINVESTAV-IPN) and the Ph. D. degree from the University of Rome "La Sapienza", Italy, in 1981, 1985 and 1992 respectively. He worked as a lecturer at the School of Electrical and Mechanical Engineering of the IPN from 1985 to 1989. From 1985 to 1995 he was working at the Automatic Control Section of the Department of Electrical Engineering of the CINVESTAV-IPN, and since 1995, at CINVESTAV-IPN, Campus Guadalajara. He has held several research stages at University of Rome "La Sapienza" and was a visiting Professor at the Laboratoire d'Automatique et d'Analyse des Systemes (LAAS) of the French Council for Scientific Research (CNR) during 19992000 and at University of Compiègne during the first semester of 2002. His main research interests include nonlinear control design, the robust regulation problem and application of control techniques to chemical processes and electromechanical devices.



\section{Guillermo Obregón-Pulido}

M.C. Guillermo Obregón Pulido was born in Guadalajara, Jalisco, México in 1974. He received the B. Sc. degree in Communications and Electronic Engineering from the University of Guadalajara (U de G), (1997) and the M. Sc. Degree from the Center of Research and Advanced Studies (CINVESTAV-IPN) (1999), where he is currently working toward his Ph. D. degree. He has been a lecturer of Control Engineering, Robotics, Physics and some other disciplines, in the Monterrey Institute of Technology and Advanced Studies. His main research interests include nonlinear control design, the robust regulation problem and application of control techniques to electromechanical system, electronic devices, and robotics. 